\title{
Industrial Centrifugal Compressors Design Validation, using Rapid Method Evolutionary Algorithms to Predict Map Characteristics Evaluation.
}

\author{
J. C. Statharas ${ }^{*}$ N. W. Vlachakis \\ Department of Aircraft Engineering, \\ Central Greece Technological University (TEI), \\ Psachna, Evias, 34400, GREECE.
}

\begin{abstract}
The purpose of this work is to model a radial compressor with a new method of predicting its map. This method calculates the air mass flow rate and the maximum pressure at each constant velocity curve of compressor operation. Two modeling approaches are proposed and applied to commercial compressors.
\end{abstract}

Keywords:- Centrifugal compressor, turbocharger, compressor map, compressor modeling, empirical model

\section{INTRODUCTION}

\subsection{Air compressors}

Air compressors are mechanical devices used to compress air for many technical applications. Two of the main categories of air compressors are the axial and radial (or centrifugal) compressors. Axial and centrifugal compressors transfer energy from a rotating device to the air (or a gas), using shaft torque to impart the moment to the gas.

Axial compressors are mainly used in large scale turbomachine applications such as in the power production industry and the aviation and marine propulsion.

\subsection{Centrifugal compressors}

Radial (or centrifugal compressors) on the other hand have a wider use in small (also in micro) or medium scale applications such as in internal combustion supercharging or turbocharging and aviation propulsion (eg. Turboshafts).

Really a large market exists for the internal combustion engine turbocharger, which is used in diesel and gasoline engines. The internal combustion engine turbocharger compresses the gas entering the manifold of the engine and is driven by a turbine running on the engine exhaust gases. In turbocharging a wide range of stable operation is necessary. This is an advantage of a centrifugal compressor with its generally wider operational area. Other applications of the compressor are for ventilation, process and chemical industries.

In the majority of cases, the so-called radial flow impeller is used. The main reasons for establishing this technology are the simplicity and robustness of the construction, the low weight and size, and finally, with even a single stage, that is, only a compression step, a satisfactory high pressure ratio that meets the turbocharging needs is achieved.

The performance of centrifugal compressors is due to the fast or very fast rotation of the impeller. The impeller rotation gives both kinetic energy and pressure to the air induced. The diffuser, as it guides the air peripherally to the spiral shell, slows it down by increasing its pressure. The diffuser may have fixed vanes or consist of single parallel walls. The spiral shell is a duct with continuously growing cross section. The duct surrounds the diffuser in the form of a spiral and collects the air from all the peripheral positions of the diffuser, leading it to a volute. The volute function is to duct flow away and to decelerate the flow further until the exit of the compressor. A detailed presentation of the main parts of the compressor is given in section 2 .

\subsection{Compressor map}

It is very useful for design purposes to know the behavior of the compressor during steady state operation. The steady state performance of the compressor is often specified in the form of a compressor map by curves that relate the rotational speed, the pressure rise across the compressor and the mass flow through the compressor. Examples of compressor maps are shown in section 3. Compressor maps usually are provided by the manufactures. Although compressor maps contain plenty of information for the compressor behaviour it is difficult for use in analytical studies. So modelling aproaches are necessary.

\subsection{Compressor modeling}

Compressor modeling is essential for predicting engine performance correctly. To get correct engine performance one has to understand how the compressor works and the amount of air it provides to the engine's cylinders for combustion. If compressor's performance is incorrectly modeled, the amount of air is wrong and the engine output torque may be wrong due to incorrect air-to-fuel ratio and combustion efficiency. Moreover uncertainty issues arise in fuel economy and in the amount of emissions in the environment. Therefore, compressor's performance has a crucial impact on engine combustion, fuel consumption, 
emmisions to the environment and output torque. It is imperative therefore to build a precise compressor model ensuring the accuracy of the whole engine model.

Usually, the static compressor map provided by the manufacturer is not in a form that is useful in engine system modeling. In literature, two distinct ways of modeling compressors can be found:

The first way is to store the compressor performance data into lookup tables and use mathematical algorithm to interpolate data.

The second one is to construct fitting functions using the experimentaly derived data to recreate the compressor's performance map. This method has been widely used in many works [1-11] giving acceptable prediction accuracy. Of course a vast range of modeling approaches and complexity levels exists, where some models are especially designed for a certain purpose, while others are meant to be more general. An extended review of model families is given in [12].

This paper mainly focuses on the fitting function method (second method) for the reason that the compressor map developed by interpolation is not always appropriate for extrapolation purposes. For instance, the regions which are not covered by the performance map can not be estimated by interpolation. Moreover the majority of the existing models are valid for the compressor used for validation, not any compressor. The proposed model has a more universal use as can be applied to any compressor.

In this study, the models of compressor flow and efficiency are fitted based on exponential function and polynomial function, respectively. Results are compared with the compressor experimental data to verify the performance of the model. In addition, the flow models derived by exponential fitting function and interpolation are compared leading to the conclusion that the fitting function methodology surpasses the interpolation by providing better extrapolation capability. We do not underestimate the excellent work done by other researchers but we feel proud for the universality of our model. Section 4 of this work presents the results of the proposed models.

\section{THE GEOMETRY OF A RADIAL COMPRESSOR}

The radial compressor consists of the compression wheel (or impeller) and the housing (or shell). These two parts constitute the rotating and stationary part of the compressor and work together to compress the introduced air and to move it radially by means of its internal configuration. The impeller main characteristics are shown in the following Fig.2.1.

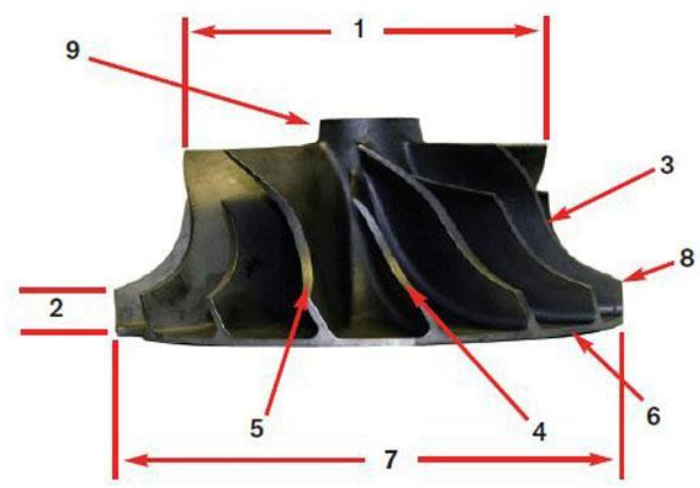

Fig. 2.1. Impeller main characteristics. 1. Inlet diameter $\mathrm{d}_{1}, 2$. Tip height $\mathrm{b}_{2}$, 3.Main blade shape, 4. Intermediate small blade (Splitter), 5. Main blade, 6. Rear wheel side, 7 . Outlet diameter $\mathrm{d}_{2}, 8$. Outlet tip edge, 9. Impeller nose.

More details of an impeller are shown in the cross section figured in Fig.2.2.

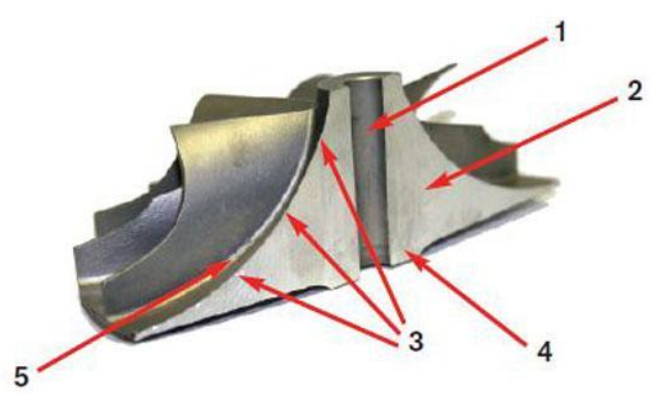

Fig. 2.2 .Impeller cross section. 1.Central hole for the operating shaft, 2.Blade basis, 3.Blade slope for air movement, 4.Impeller rear side extension, 5.Blade root

There are several types of impeller designs. Each has its advantages and its corresponding use.

Pure radial design (Fig.2.3) is not used today because it does not perform well despite the high speeds achieved. If it is used with a vaned-type diffuser, the efficiency is increased but it lacks in air mass flow rate and is not suitable for the automotive industry requiring a wide range of revolutions. It is used in diesel engines and generators operating at a limited number of revolutions.

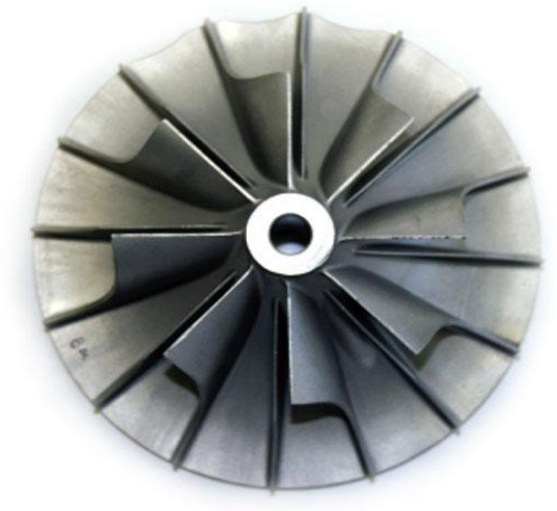

Fig. 2.3 . Pure radial impeller 
Impellers with only single normal blades (Fig.2.4) are rarely seen today and only in applications with lower rotation speeds. There are not suitable for applications with high performance and high compression. They lack in the air supply at high speeds despite the good performance and high pressure that achieves.

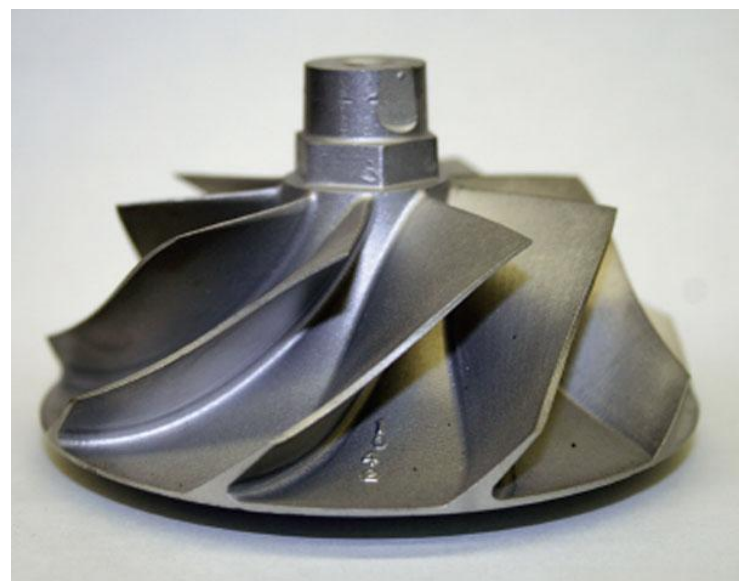

Fig. 2.4. Impeller wheel with normal blades.

Impellers with intermediate small blades (splitters) (Fig.2.5) in between the main blades, appear more frequently today because at high speeds the vacuum created helps to increase the air mass flow rate. That is, the small blade helps to manage and compress the air as it is accelerated according to the wheel rotation.

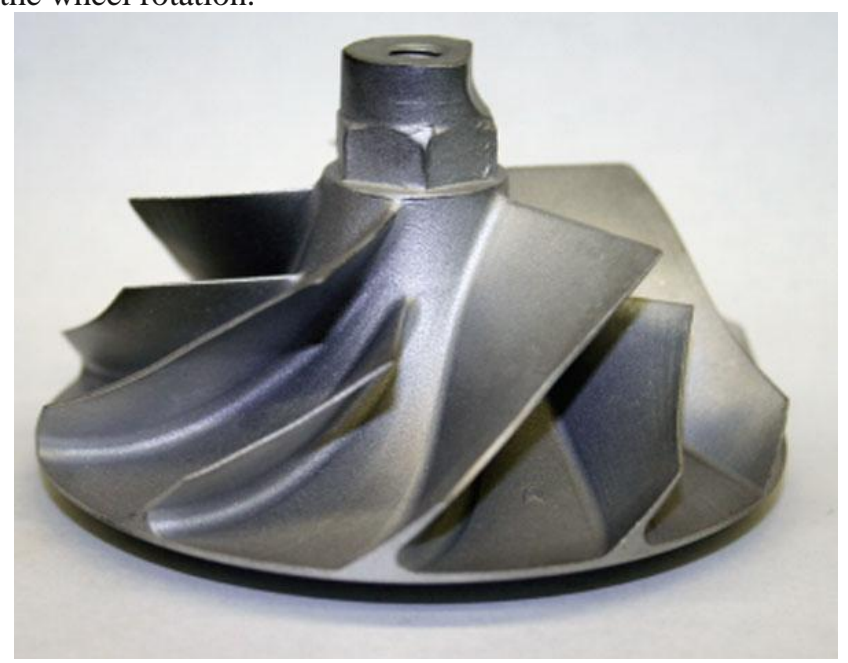

Fig. 2.5 Impeller wheel with splitters

Backward leaned blades (or vanes) (Fig.2.6) are the most common in today's design. They allow the compressor to operate at a greater range of revolutions that is necessary in high performance vehicle engines since air diffusion begins before entering the diffuser.

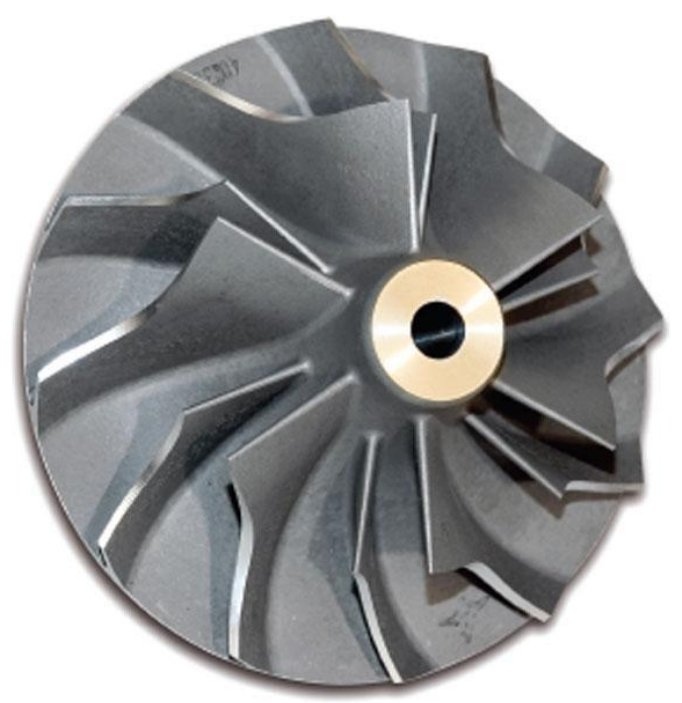

Fig.2.6.Impeller with backward leaned vanes.

There are vanes that are fully or partially backward leaned (Fig. 2.7). Fully backward leaned vanes are those that have a reversed curve across the diameter of the impeller. Partially backward leaned vanes were devised to reduce the weight of the wheel so as to be faster on accelerations. But they are not used because they are not so effective.

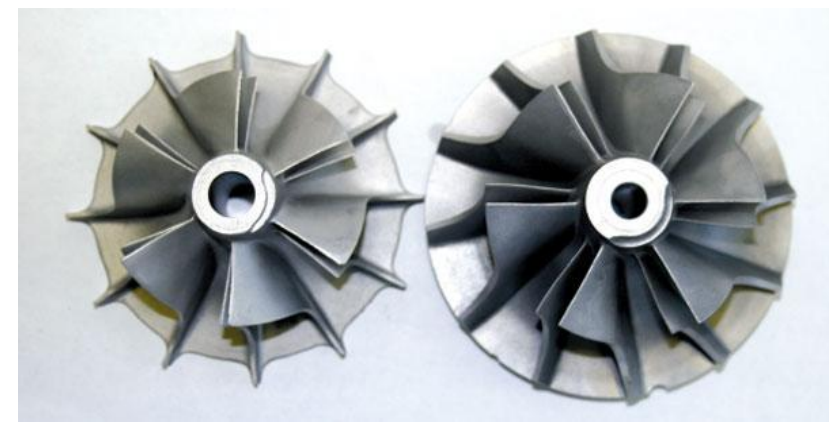

Fig. 2.7 Fully or partially backward leaned vanes

Another feature of the compressors is the trim. To understand the definition of trim we need to know the inflow diameter (inducer) and the outflow diameter (exducer) of the compressor. More specifically trim is defined as the area ratio:

$$
\text { trim }=\left(\frac{\text { inducer area }}{\text { exducer area }}\right)^{2} * 100=\left(\frac{d_{1}}{d_{2}}\right)^{2} * 100
$$

The compressor housing (or shell) creates the internal space in which the air moves (Fig.2.8). 


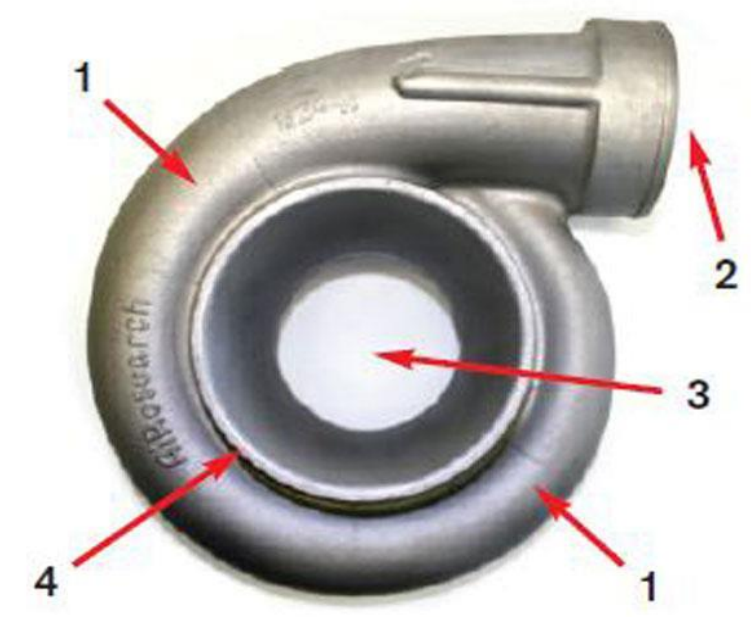

Fig. 2.8. Compressor housing.1. Spiral, 2. Compressor outlet, 3. Inlet surface, 4. Inner connection diameter

At the end of the blade we see the diffuser (Fig.2.9). The shape of the diffuser helps to convert the air velocity to pressure.

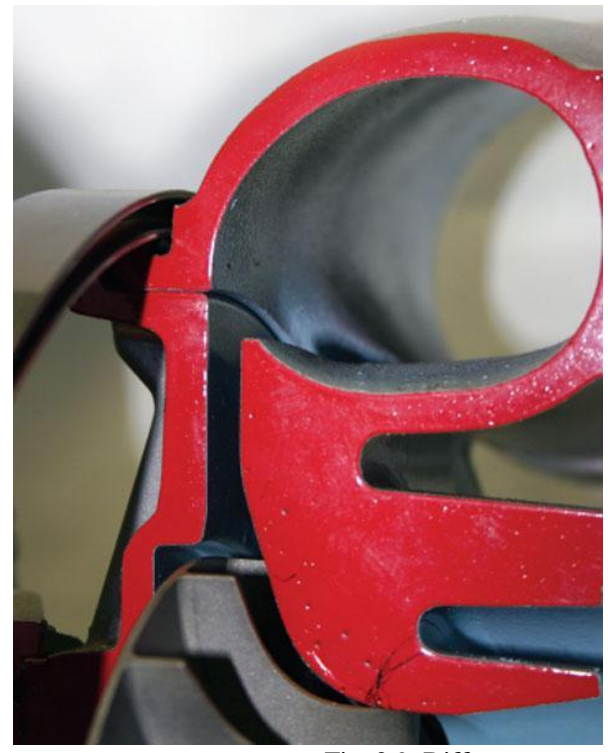

Fig. 2.9. Diffuser

In some cases the diffuser has blades (see Fig. 2.10). This helps to increase the efficiency but also greatly reduces the air mass flow rate operating range. So it will only be used in lowspeed engines.

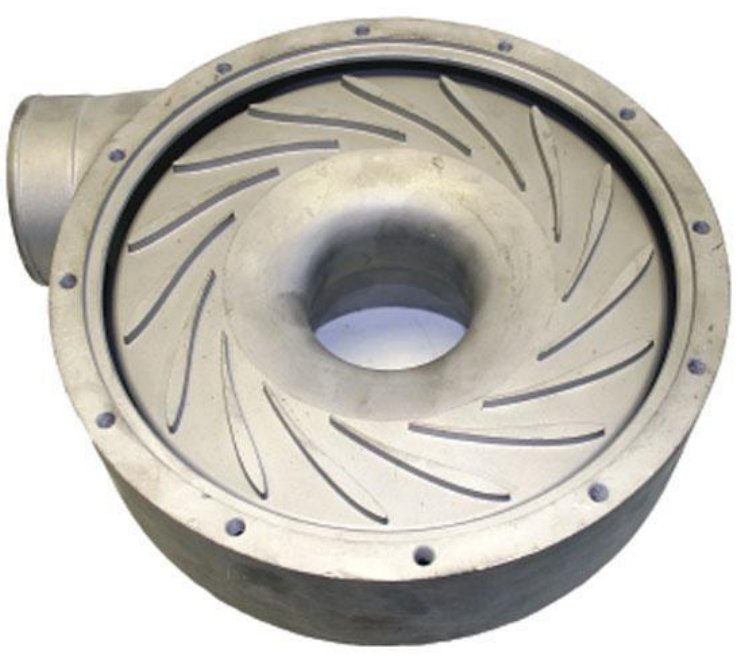

Fig. 2.10. Diffuser with blades

Another feature of the turbo compressors is the A/R ratio. It is the ratio of the surface area of the cross section of the spiral that is called A, to the distance defined by the center of the impeller to the radius of area A and is called R (Fig.2.11).

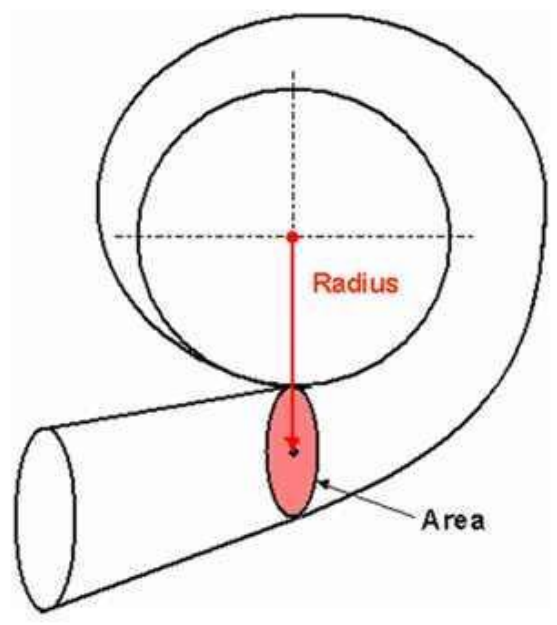

Fig. 2.11. Definition of $\mathrm{A} / \mathrm{R}$ ratio.

This parameter has a strong effect on the performance and operating characteristics of the turbocharger. The $A / R$ ratio of the compressor does not play an important role because its change does not affect valuably the whole turbocharger system. From the turbine side, however, the $A / R$ is used to adjust flow capability and matching.

On the intake side there is an opening perimetrically to the shell which allows recirculation of the air (Fig.12). This has the effect of reducing the surge problem especially at low revolutions since there is always air available for compressor operation and flow stabilization. 


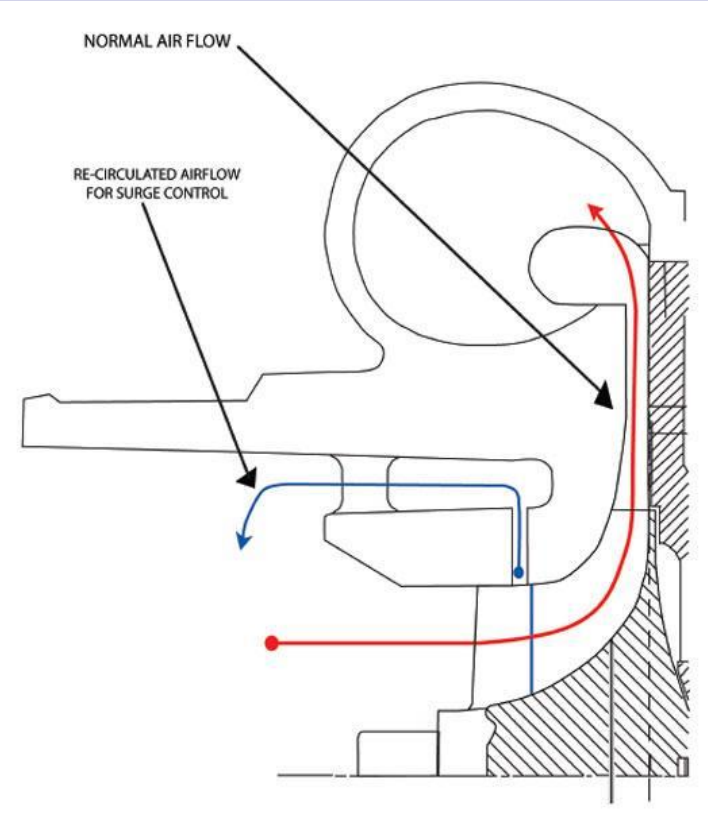

Fig. 2.12. Opening for recirculation

\section{COMPRESSOR MODELING}

There are various methods for modeling the compressor behavior which are based on the parameterization of the dimensionless air mass flow rate using several parameters. From the different parameterizations developed, a simple method has been developed in [13] and shows that a simple model gives good accuracy among the most important areas of compressor maps. The advantages of the simple model are:

- A good description of the air mass flow rate in the low-speed area, where the machines operate most of the time.

- Simple adjustment

The models are based on the parameterization of the flow factor $\Phi$ :

$$
\Phi=\frac{m_{c}}{\rho \frac{\pi}{4} d^{2}{ }_{2} U_{2}}
$$

where $\dot{m}_{c}$ is the air mass flow rate, $\rho$ is the air density and $U_{2}$ is the tip speed needed:

$$
U_{2}=\frac{\pi d_{2} N}{60}
$$

where $\mathrm{N}$ is the number of revolutions per minute (RPM).

Assuming that the flow factor can be parameterized as $\Phi$, then it is possible to invert and express the air mass flow rate as a function of $\Phi$ :

$$
\dot{m}_{c}=\left(\rho \frac{\pi}{4} d_{2}^{2} U_{2}\right) \Phi
$$

We still have to model the flow factor. There are three models for this. In all the models proposed, the basic parameter $\Psi$ and Mach-number $\mathrm{M}$ are used

$$
\Psi=c_{P} T_{a}\left(\frac{\Pi^{\frac{r-1}{\gamma}}-1}{0.5 U_{2}^{2}}\right)
$$

$$
M=\frac{U_{2}}{\sqrt{y R T_{\alpha}}}
$$

where $c_{p}$ is the air specific heat at constant pressure, $T_{\alpha}$ is the ambient air temperature, $\Pi$ is the compression ratio, $\gamma$ is the ratio of air specific heats and $R$ is the air specific gas constant.

\subsection{Compressor models}

1) According to [13], the basic parameter $\Psi$ is expressed as a function of $\Phi$ and $\mathrm{M}$ in the following way:

$$
\boldsymbol{\psi}=\frac{K_{1}+K_{2} \Phi}{K_{3}-\Phi}, K_{i}=k_{i 1}+k_{i 2} M, i=1,2,3
$$

where $K_{i}$ coefficients to be determined.

2) According to [14], $\Phi$ and $\Psi$ are expressed as a quarter of an ellipsis (Fig. 3.1), which results in the flow factor $\Phi$ being expressed only as a function of the basic parameter $\Psi$.

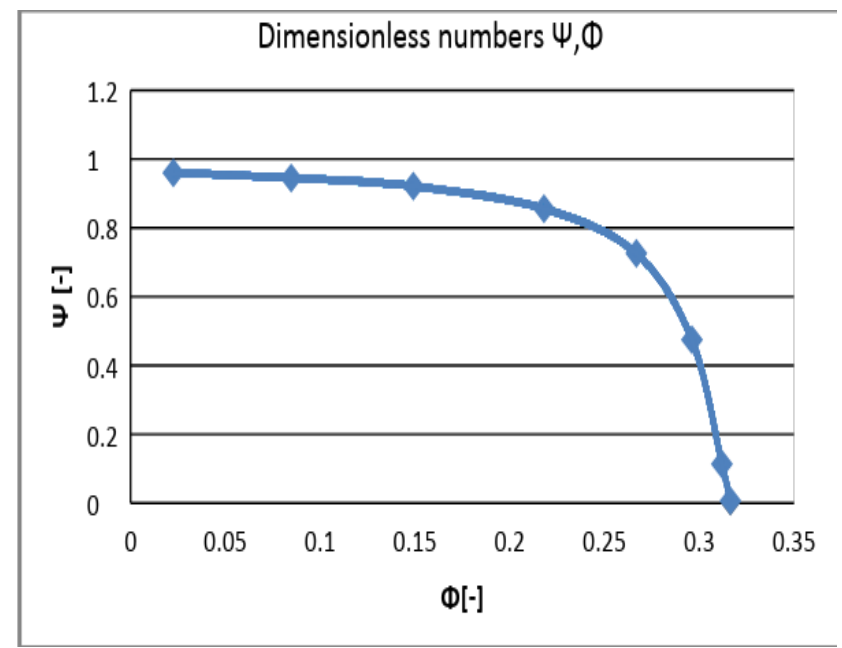

Fig. 3.1. Variation of $\Psi$ versus $\Phi$ according to [14]

3) According to [15] the flow factor $\Phi$ can also be expressed only as a function of $\Psi$ :

$$
\Phi=K_{1}+\left(1-e^{\psi^{\kappa_{2}}+K_{3}}\right)
$$

\subsection{Finding the coefficients}

Irrespectively of the method used, the coefficients, which apply only to a particular compressor, must be determined. So each time we want to model a different compressor, it is necessary to repeat the procedure of determining the coefficients again and again.

In all types, the $K_{1}, K_{2}$ and $\mathrm{K}_{3}$ parameters (if used) are determined using the least squares method in the Matlab Curve Fitting Toolbox.

$$
K_{i}=k_{i 1}+k_{i 2} M, i=1,2,3
$$

$\mathrm{K}_{1}=\mathrm{k}_{11}+\mathrm{k}_{12} \mathrm{M}$

$\mathrm{K}_{2}=\mathrm{k}_{21}+\mathrm{k}_{22} \mathrm{M}$

$\mathrm{K}_{3}=\mathrm{k}_{31}+\mathrm{k}_{32} \mathrm{M}$

We selected the Mitsubishi TD04-09B compressor for modeling its map. The main characteristics of this compressor are shown in Table 3.1 and the coressponding compressor map is shown in Fig.3.2. Below we calculate the coefficients from the Mitsubishi TD04-09B compressor map (Fig.3.2). First, from the map we find the experimental points as $\Pi\left(\mathrm{m}_{\mathrm{c}}\right)$ 
at different rotational speeds $\mathrm{N}$. Then equations (3.1), (3.2), (3.4), (3.5) are used to calculate $\Psi(\Phi)$. Table 3.2 shows an example of the results for rotational speed $\mathrm{N}=140000 \mathrm{RPM}$.

Table 3.1 Basic characteristics of Mitsubishi TD04 -09B compressor
\begin{tabular}{|c|c|c|c|c|}
\hline $\begin{array}{c}\text { Turbo } \\
\text { model: }\end{array}$ & $\begin{array}{c}\text { Compressor } \\
\text { Trim: }\end{array}$ & $\begin{array}{c}\mathbf{d}_{\mathbf{1}} \\
\mathbf{i n} /(\mathbf{m m})\end{array}$ & $\begin{array}{c}\mathbf{d}_{\mathbf{2}} \\
\mathbf{i n} /(\mathbf{m m})\end{array}$ & $\begin{array}{c}\text { Compressor } \\
\text { Diameter } \\
\text { Average: }\end{array}$ \\
\hline $\begin{array}{l}\text { Mitsubishi } \\
\text { TD04-09B }\end{array}$ & 50 & $\begin{array}{c}1.365 \\
(34.671)\end{array}$ & $\begin{array}{c}1.930 \\
(49.022)\end{array}$ & 1.6475 \\
\hline
\end{tabular}

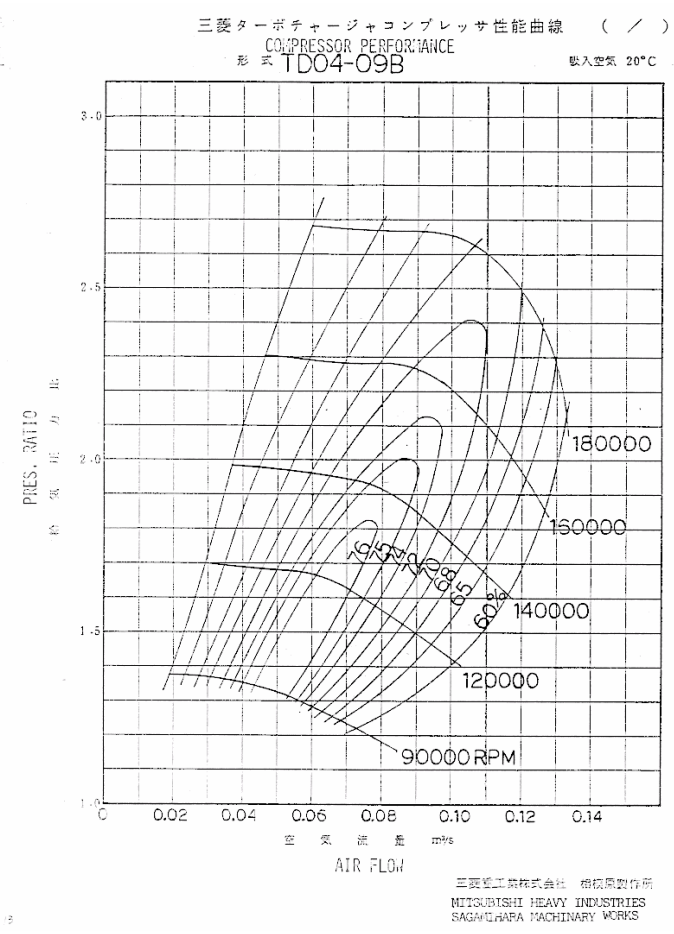

Fig. 3.2. Mitsubishi TD04-09B compressor map

\begin{tabular}{|r|r|r|r|}
\hline \multicolumn{5}{|c|}{ Table 3.2 (example N=140kRPM) } \\
\hline \multicolumn{3}{|c|}{ rotational speed N=140000 RPM } \\
\hline $\mathrm{U}_{\mathrm{c}=359,17 \mathrm{~m} / \mathrm{sec}}$ & \multicolumn{2}{|c|}{$\mathrm{M}=1,0345$} \\
\hline 1,986 & $\mathrm{~m}_{\mathrm{c}}$ & 0,044396 & 1,007288 \\
\hline 1,982 & 0,037 & 0,056395 & 1,004029 \\
\hline 1,973 & 0,053 & 0,063595 & 0,996681 \\
\hline 1,963 & 0,060 & 0,071994 & 0,988487 \\
\hline 1,949 & 0,067 & 0,080393 & 0,976966 \\
\hline 1,94 & 0,072 & 0,086393 & 0,969528 \\
\hline 1,931 & 0,076 & 0,091193 & 0,962066 \\
\hline 1,898 & 0,083 & 0,099592 & 0,934489 \\
\hline 1,87 & 0,087 & 0,104391 & 0,910821 \\
\hline 1,837 & 0,091 & 0,109191 & 0,882599 \\
\hline 1,794 & 0,096 & 0,115191 & 0,845277 \\
\hline 1,752 & 0,101 & 0,12119 & 0,8082 \\
\hline 1,695 & 0,107 & 0,128389 & 0,756852 \\
\hline 1,629 & 0,114 & 0,136789 & 0,695832 \\
\hline 1,61 & 0,116 & 0,139189 & 0,677939 \\
\hline & & & \\
\hline & & & \\
\hline
\end{tabular}

From the calculated $\Psi(\Phi)$ using the Matlab Curve Fitting Toolbox (Table 3.3) we find the coefficients $K_{1}, K_{2}$ and $K_{3}$ for different rotational speeds $\mathrm{N}$ (Table 3.4). For example, the coefficients for the method in [13] are shown in Fig. 3.3. We see from Figure 3.3 and in Table 3.3 the function $\mathrm{f}(\mathrm{x})=\left(\mathrm{a}+\mathrm{b}^{*} \mathrm{x}\right) /(\mathrm{c}-\mathrm{x})$ corresponds to Eq.(3.6) and $\mathrm{a}, \mathrm{b}, \mathrm{c}$ are the coefficients $\mathrm{K}_{1}, \mathrm{~K}_{2}$ and $\mathrm{K}_{3}$.

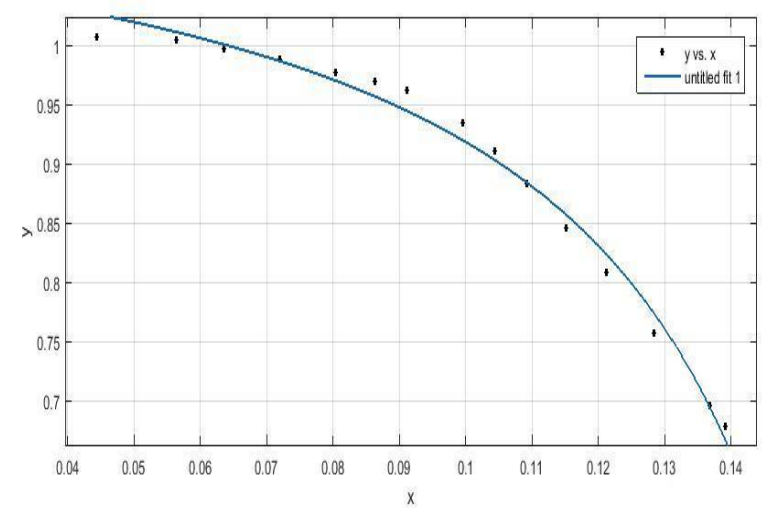

Fig. 3.3. $K_{1}, K_{2}$ and $K_{3}$ coefficients for the method in [13].

Table 3.3 matlab curve fitting equation output

General model: $f(x)=(a+b * x) /(c-x)$

Coefficients (with 95\% confidence bounds):

$$
\begin{aligned}
& a=0.1917(0.1715,0.2119) \\
& b=-1.182(-1.253,-1.11) \\
& c=0.1801(0.1651,0.1951)
\end{aligned}
$$

Table 3.4

\begin{tabular}{|c|c|c|c|c|}
\hline \multicolumn{5}{|c|}{ Jensen [13] } \\
\hline $\mathrm{N}$ & $\mathrm{M}$ & $\mathrm{K}_{1}$ & $\mathrm{~K}_{2}$ & $\mathrm{~K}_{3}$ \\
\hline 90000 & 0,66504 & 0,2541 & $-1,483$ & 0,2176 \\
\hline 120000 & 0,88672 & 0,2194 & $-1,295$ & 0,1978 \\
\hline 140000 & 1,0345 & 0,1916 & $-1,181$ & 0,18 \\
\hline 160000 & 1,18229 & 0,3534 & -2.458 & 0,1493 \\
\hline 180000 & 1,33008 & 0,1867 & $-0,9549$ & 0,1991 \\
\hline
\end{tabular}

The next step is to find the coefficients $\mathrm{k}_{\mathrm{ii}}$ using the equation (3.8) that depend on the number M. The least squares method of Matlab is used. The final coefficients for all methods are shown in Table 3.5 . 
Table 3.5 The coefficients of equations

\begin{tabular}{|c|c|c|c|c|c|c|}
\hline Method & $\mathrm{k}_{11}$ & $\mathrm{k}_{12}$ & $\mathrm{k}_{21}$ & $\mathrm{k}_{22}$ & $\mathrm{k}_{31}$ & $\mathrm{k}_{32}$ \\
\hline$[13]$ & 0,3215 & $-0,10135$ & $-2,0111$ & 0,794088 & 0,2361 & $-0,02782$ \\
\hline (Ellipsis) [14] & 59,78 & $-35,261$ & 0,587 & 0,302388 & - & - \\
\hline$[15]$ & $\begin{array}{c}786,45 \\
3\end{array}$ & 1319,45 & 0,970275 & 2,528758 & 1,757268 & 4,14 \\
\hline
\end{tabular}

In [15], the $\mathrm{k}_{\mathrm{i}}$ coefficients must be carefully calculated especially at high speeds ( $>140000$ RPM) because in exponential terms when rounding can easily result in incorrect results. This method is proposed in large diameter turbocompressors. $\mathrm{d}_{2}>0.5 \mathrm{~m}$.

To calculate air mass flow rate and compression ratio according to each method, the following equations are used:

$$
m_{c}=\Phi \rho_{a} \frac{\pi}{4} d_{2}^{2} U_{2}
$$

$$
\Pi=\left[\frac{0,5 U_{2}^{2} \Psi}{c_{p} T_{a}}+1\right]^{\frac{\gamma}{r-1}}
$$

Figure 3.4 shows the results of the three methods applied to the TD04-09B compressor.

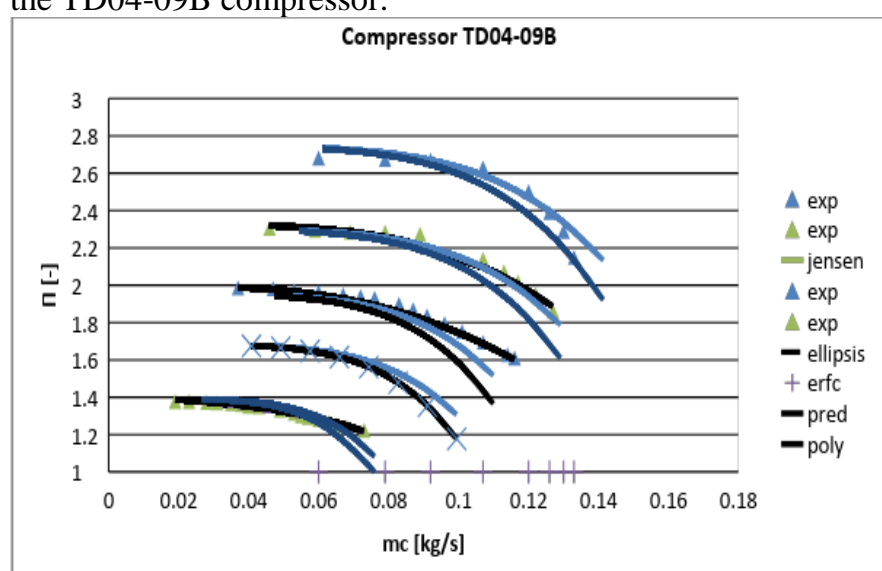

Fig. 3.4. Intercomparison of the modeling methods

The efficiency $n_{c}$ can also be expressed as a function of $\Phi$ and $\mathrm{M}$ in the following way:

$$
n_{c}=a_{1} \Phi^{2}+\alpha_{2} \Phi+\alpha_{3}, \alpha_{i}=\frac{\alpha_{i 1}+a_{i 2} M}{a_{i 3}-M}
$$

Figure 3.5 shows the results of the efficiency $n_{c}$ for the TD04-09B compressor.

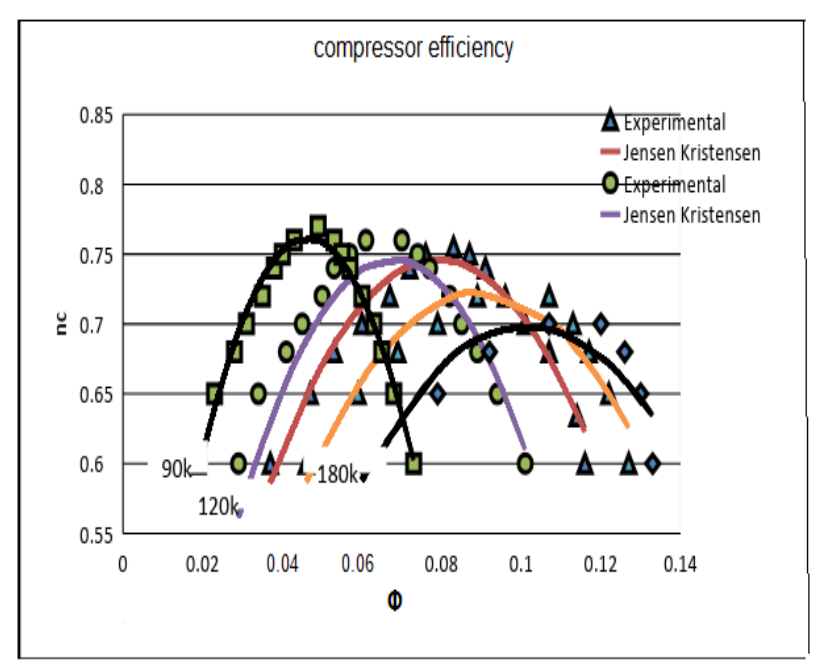

Fig. 3.5. TD04-09B Compressor efficiency

\section{AD-HOC MODELING OF THE RADIAL COMPRESSOR MAP.}

It would be very useful if we could, by using some equations, to determine the characteristic constant velocity curves of the compressor map. It would also be even more useful if this resulted from simple geometric characteristics of the compressor and applied to a wide range of compressor sizes. In the preceded methods the modeling of compressor maps and the calculation of respective coefficients are made for each compressor. In our own methodology, the calculated coefficients apply to all compressors.

\subsection{Statharas-Vlachakis calculational procedure}

The calculation of a minimum and a maximum value of the air mass flow rate and the calculation of the maximum compression ratio of the compressor will be made. The calculation is based on empirical equations and geometric characteristics of the compressor.

The minimum value of air mass flow rate is:

$$
m_{\min }=0,08 \cdot c_{r 1} \cdot \frac{\pi d_{1}^{2}}{4} \cdot z^{0,4}
$$

The radial inlet velocity is:

$$
c_{r 1}=\frac{c_{u 1}}{\tan a_{1}}
$$

The peripheral component of the absolute input velocity is:

$$
c_{u t}=u_{1} \cdot \psi
$$

The peripheral velocity is:

$$
u_{1}=\frac{\pi * d_{1} * N}{60}
$$

The load factor is : 


$$
\psi=\frac{1}{\frac{\tan \beta_{2}}{\tan a_{2}}+\frac{1}{\sigma}}
$$

The Weisner slip factor:

$$
\sigma=1-\frac{\sqrt{\cos \beta_{1}}}{z}
$$

where $\mathrm{z}$ is the number of blades

$\alpha_{1}$ inlet flow angle

$\alpha_{2}$ outlet flow angle

$\beta_{1}$ inlet blade angle

$\beta_{2}$ outlet blade angle
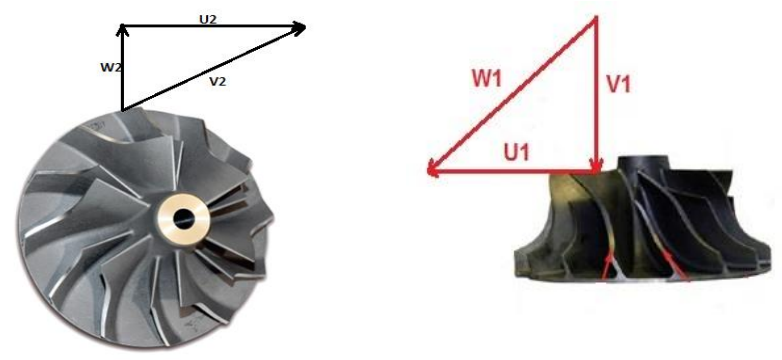

Fig. 4.1. Inlet-outlet compressor velocities

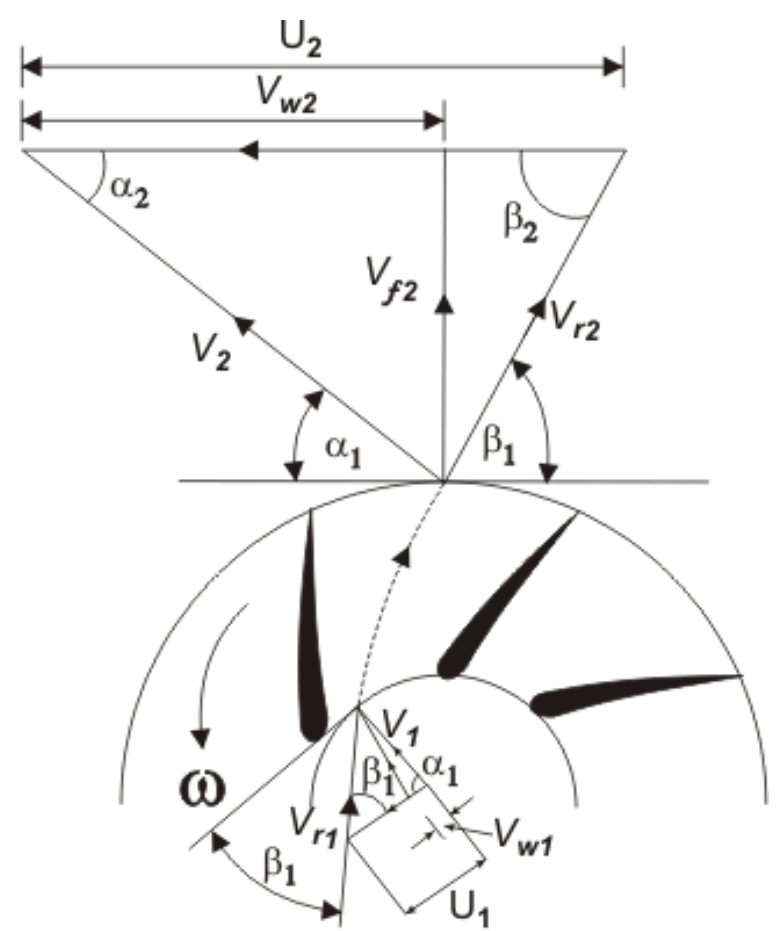

Fig. 4.2. Inlet-outlet compressor angles

The maximum mass flow rate is given by the formula : $m_{\max }=a \cdot c_{r 2} \cdot \frac{\pi d_{2}^{2}}{4} \cdot z^{0,4}$
The blade blockage factor of diameters ratio is :

$$
a=\left(0,53 \frac{d_{1}}{d_{2}}-0,208\right) \cdot\left(\frac{d_{2}}{d_{0}}\right)^{0,05}
$$

where $\mathrm{d}_{0}=0,032 \mathrm{~m}$.

A good estimation of the radial exit velocity is suggested:

$$
c_{r 2}=0,36 \cdot u_{2}
$$

The peripheral outlet velocity is:

$u_{2}=\frac{\pi \cdot d_{2} \cdot N}{60}$

The maximum pressure ratio of the compressor is :

$$
\Pi_{\max }=c \cdot\left(1+0,42\left(\gamma_{\alpha}-1\right) \cdot M^{2}\right)^{\frac{\gamma_{\alpha}^{-}-1}{V_{\alpha}}}
$$

Where $c$ is the correction coefficient for the outlet diameter $\mathrm{d}_{2}$ :

$c=4,17 \cdot d_{2}+0,866$

and the tip Mach number:

$$
M=\frac{u_{2}}{\sqrt{1 R T_{a}}}
$$

\subsection{Determining the compressor map}

Depending on the mass flow rate, the curves of the compressor map will be derived according to the following empirical equation:

$$
\Pi_{\text {erf }}(m)=1,03 \cdot \Pi_{\max } \cdot \operatorname{erfc}\left[0,2 \cdot\left(\frac{\dot{m}}{\dot{m}_{\max }}\right)\right]^{2 \cdot e}
$$
[16].

The above equation uses the complementary error function

Similarly, in relation to the mass flow rate, the curves of the compressors map can also be obtained according to the following empirical equation

$$
\Pi_{\text {poly }}(m)=1,02 \cdot \Pi_{\max } \cdot\left[1-0,3 \cdot\left(\frac{m_{i}}{m_{\max }}\right)\right]^{2 \cdot e}
$$

The ratio of the masses $\dot{m}_{i} / \dot{m}_{\max }$ takes intermediate values between $i=\min$ and $i=\max$. The coefficient of compressor morphology in both equations is given by the equation:

$$
e=27,78 d_{2}+1,111
$$

\subsection{Application-Examples}

To confirm this methodology we will apply it to various commercial compressors that cover the entire range of compressors that can be used in piston combustion engines. The compressors used have the following characteristics as shown in Table 4.1.

\begin{tabular}{|c|l|c|c|c|}
\hline & Compressor & $\begin{array}{c}\text { Tnlet } \\
\text { diameter } \mathrm{d}_{1} \\
(\mathrm{~mm})\end{array}$ & $\begin{array}{c}\text { Outlet } \\
\text { diameter } \mathrm{d}_{2} \\
(\mathrm{~mm})\end{array}$ & trim \\
\hline 1 & Garrett gt06_50 & 22,63 & 32 & 50 \\
\hline 2 & Garrett gt12_50 & 29 & 41 & 50 \\
\hline 3 & Garrett gt15_56 & 32,9 & 43,9 & 56 \\
\hline 4 & Garrett gt17_49 & 34,3 & 49 & 49 \\
\hline 5 & Mitsubishi td04-09b & 34,7 & 49 & 50 \\
\hline 6 & Garrett gt25_550 & 48,4 & 60 & 65 \\
\hline 7 & Garrrett gtx3077r & 58 & 76 & 58 \\
\hline 8 & Garrett gt4202r & 75,2 & 102 & 54 \\
\hline 9 & Holset hx82 & 98 & 141 & 48 \\
\hline
\end{tabular}


The figures 4.3-4.10 show the compressor maps predicted by using the new method. In the figures the two procedures are shown as erfc and poly. As we can see, the approach with the new prediction method is very good, since the constant velocity curves created are close to the actual operating points of the compressors given by the manufacturers. Particularly in the garrett gt06-50, gt17-49 and gt12-50 compressors the approximation of the air mass flow rate constant velocity curves but also the pressure ratio of the compressor is very good.

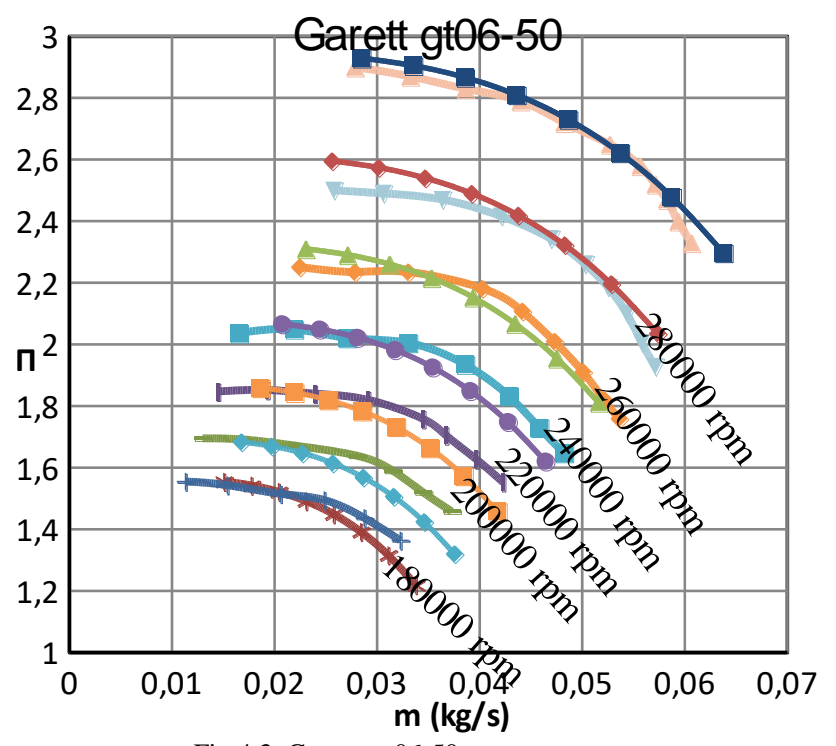

Fig.4.3. Garrett gt06-50 compressor map

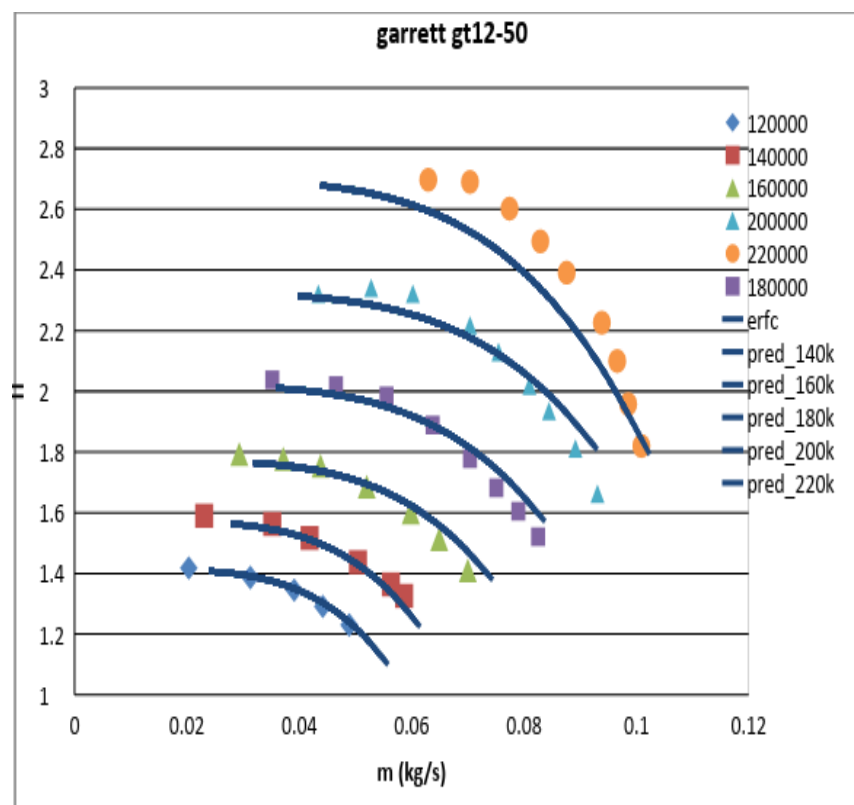

Fig.4.4. Garrett gt12-50 compressor map garrett gt17 49

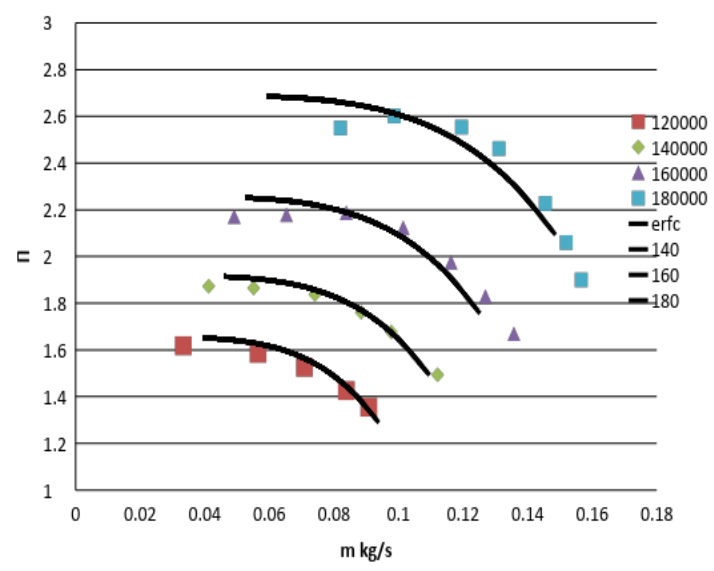

Fig.4.5. Garrett gt17 49 compressor map

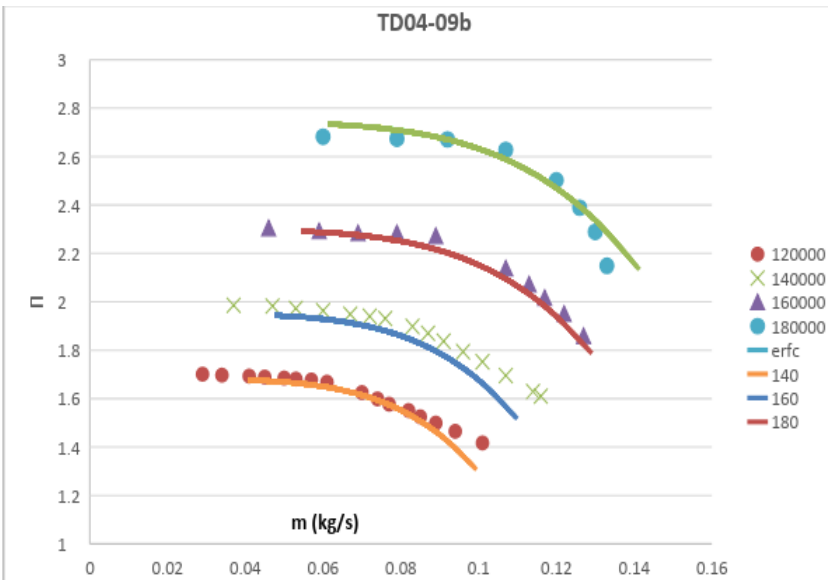

Fig.4.6.Mitsubishi td04-09b compressor map

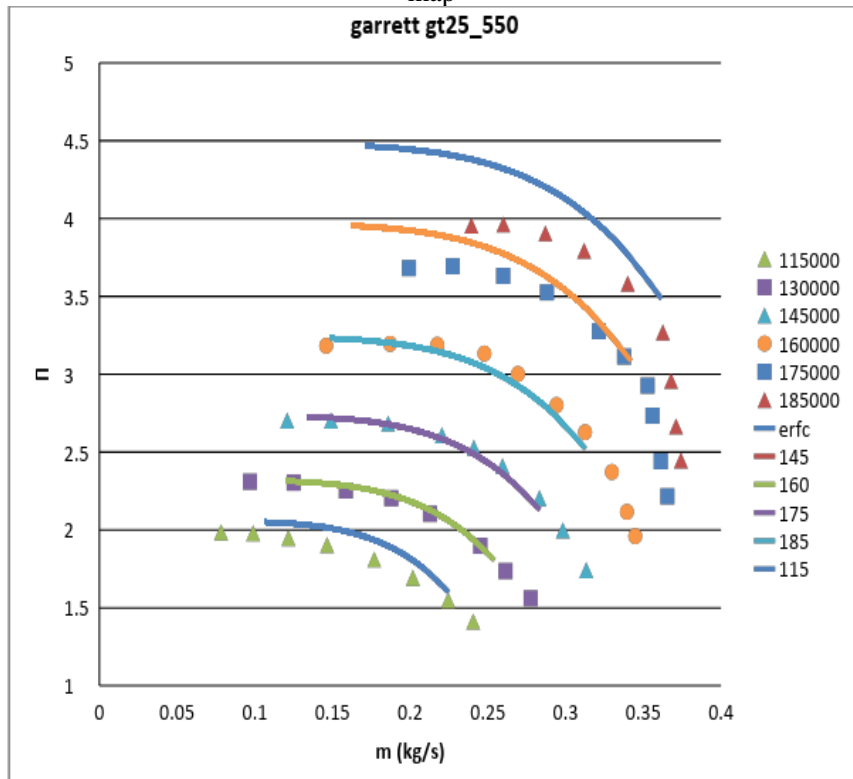

Fig.4.7. Garrett gt25_550 compressor map 


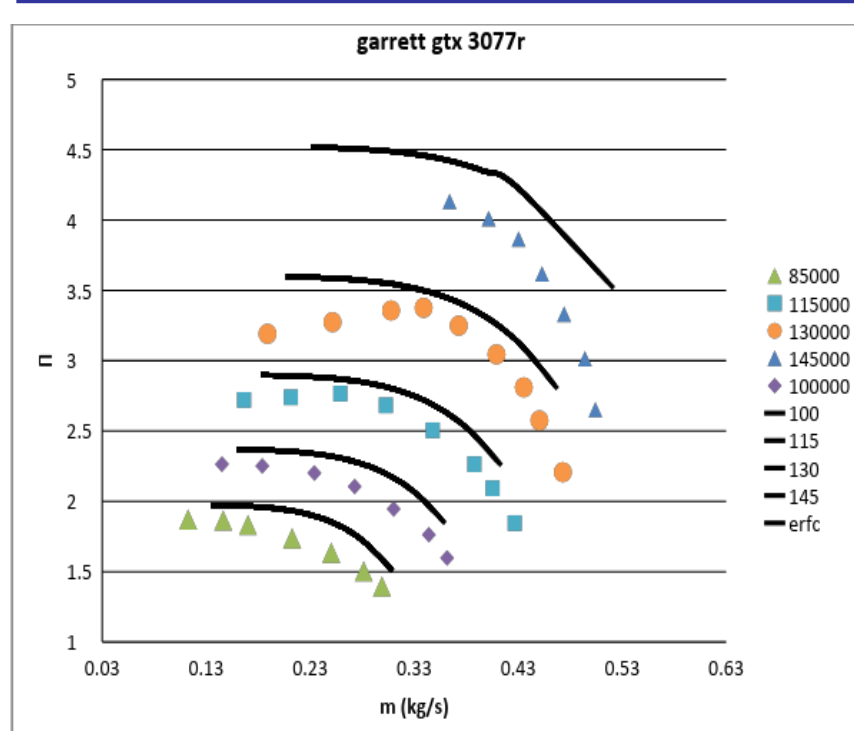

Fig.4.8. Garrett gtx3077r compressor map

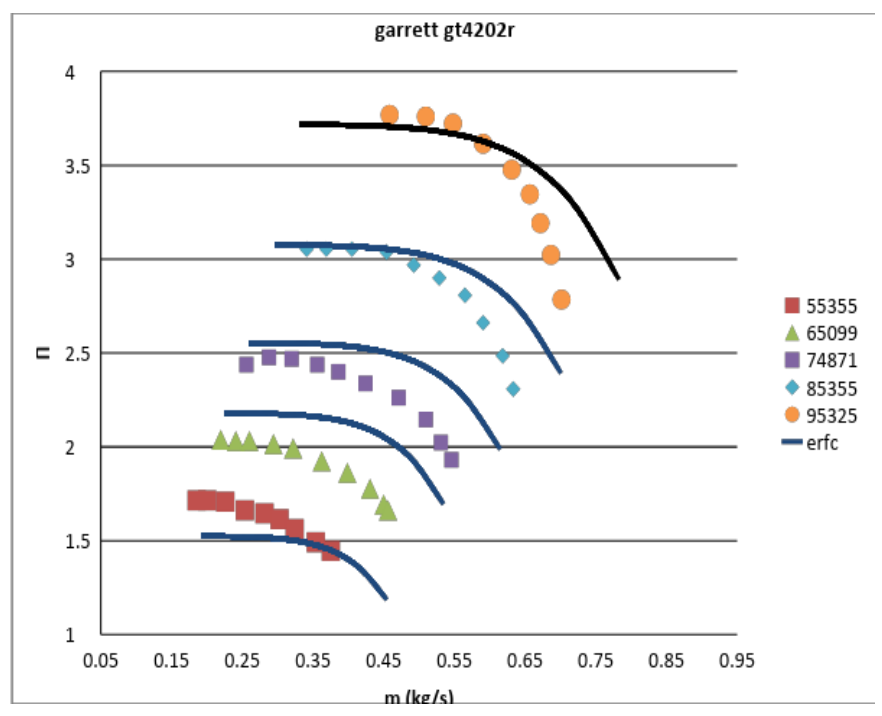

Fig.4.9. Garrett gtx $4202 \mathrm{r}$ compressor map

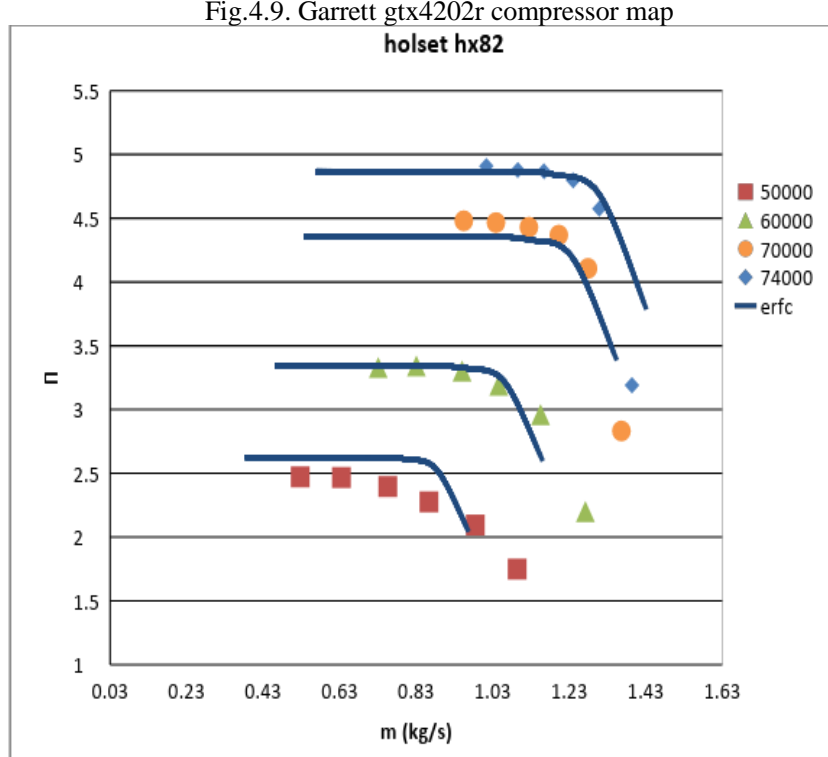

If we compare the new methodology with the old ones (Fig. 4.11) we see that we have a very good approach with respect to the older modeling approaches. Let us note that the older models do not calculate the air mass flow rate not the pressure compression ratio.

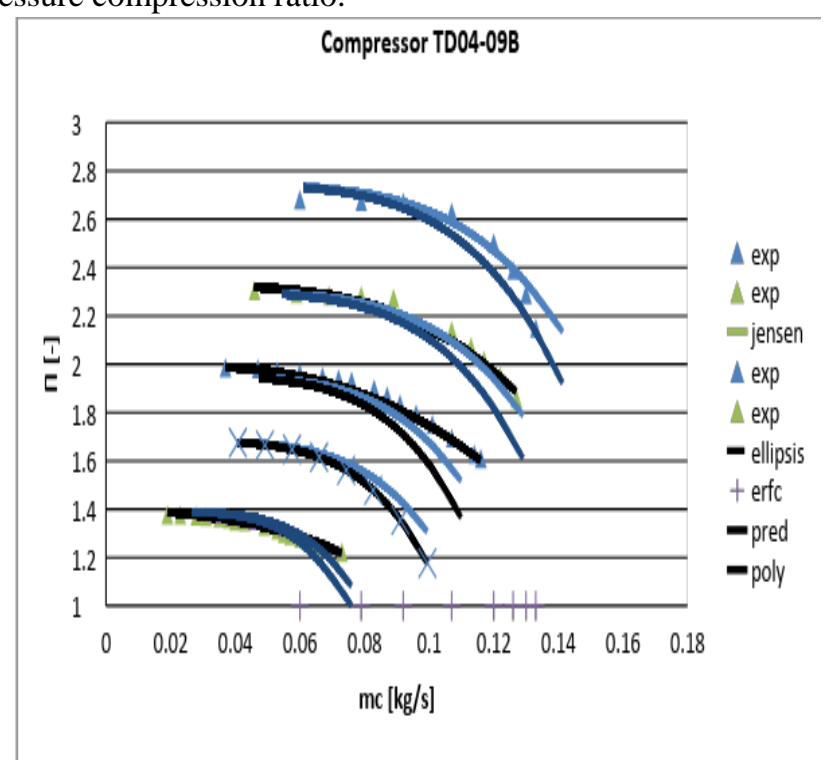

Fig. 4.11. Intercomparison of compressor map prediction methodologies

\subsection{Particularities of compressors}

When choosing the right compressor we have to take account of the trim. That is, the larger the ratio of the diameters square of the impeller, the greater the air mass flow rate that compressor can support.

$$
\text { trim }=\left(\frac{d_{1}}{d_{2}}\right)^{2} * 100
$$

Compressors with large inlet to small outlet diameter will support large amounts of air at low pressure as opposed to a small inlet to large outlet diameter compressor that supports less mass flow rate at relatively higher pressures.

Fig.4.10. Holset hx82 compressor map 

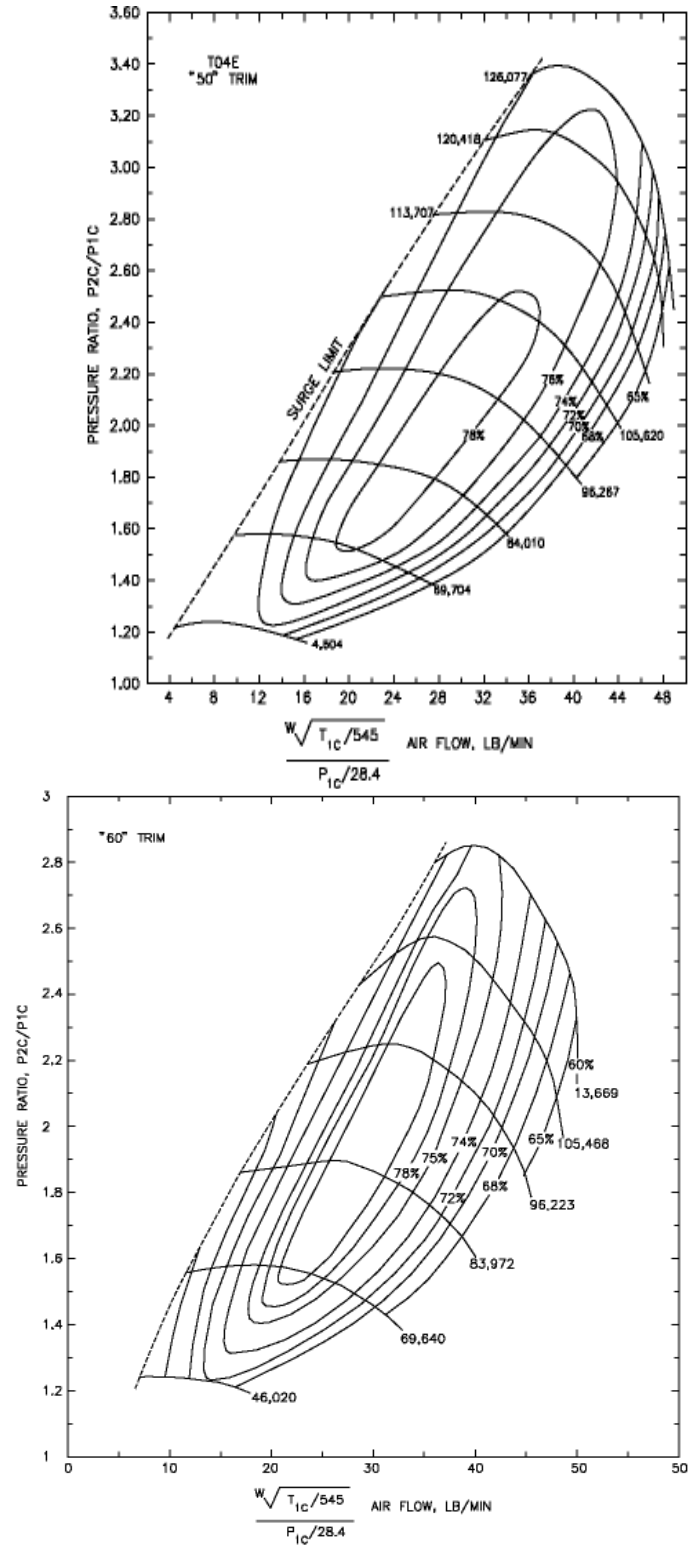

Fig. 4.12. Compressor maps having different trim

In the two compressor maps (Figure 4.12) it is evident that although having the same outlet diameter $\mathrm{d}_{2}$ the larger trim '60' compressor has higher mass flow rates at lower pressures. For example, the first compressor for $\mathrm{P}_{2} / \mathrm{P}_{1}=2.2$ has an air mass flow rate of up to $44 \mathrm{lb} / \mathrm{min}$ while in the second compressor for the same pressure ratio the air mass flow rate is up to $50 \mathrm{lb} / \mathrm{min}$.

\section{CONCLUSIONS}

The work presented in this paper address the issue of modeling the compressor performance map, proposing an error function based model for flow rate. The models are validated by comparing with the compressor performance map provided by the manufacturer. Results show that the performance predicted by the models is closely comparable with the real performance map. Moreover the error function based flow rate model shows credible extrapolation capability which the interpolation methodology does not have. The compressor model can be applied to mean value model of turbocharged diesel engine for non-linear control and state estimation.

\section{REFERENCES}

[1] L. Eriksson, L. Nielsen, J. Brugård, J. Bergström, F. Pettersson, and Per Andersson, "Modeling of a turbocharged SI engine", Annual Reviews in Control, 26(1):129-137, 2002

2] .T. Gravdahl, O. Egeland, and S.O. Vatland, "Active surge control of centrifugal compressors using drive torque", vol.2 of Proc. IEEE Conf. on Decision and Control, 2001.

[3] L. Guzzella and A. Amstutz, "Control of diesel engines", Control Systems Magazine, IEEE,18(5):53-71, Oct 1998.

[4] L. Guzzella and A. Amstutz, "Control of diesel engines", Control Systems Magazine, IEEE,18(5):53-71, Oct 1998

[5] F.K. Moore and E.M. Greitzer, "A theory of post-stall transients in axial compression systems: Part i - development of equations", Trans. of ASME, J. of Engineering for Gas Turbines and Power, (108):68-76, 1986. .

[6] P. Moraal and I. Kolmanovsky. Turbocharger modeling for automotive control applications. In SAE World Congr., Techn. Paper 1999-01 0908, March 1999.

[7] M. Müller, E. Hendricks, and Spencer C. Sorenson, "Mean value modelling of turbocharged spark ignition engines", In SAE World Congr., Techn. Paper 980784, Detroit, USA, 1998.

[8] A. Plianos and R. Stobart, "Modeling and control of diesel engines equipped with a two-stage turbo-system", In SAE World Congr. Techn. Paper 2008-01-1018, 2008

[9] A. Thomasson and L. Eriksson, "Modeling and control of co-surge in bi-turbo engines", In Proc. of IFAC World Congr., pages 1301013015, 2011.

[10] J. Wahlström and L. Eriksson, "Modelling diesel engines with a variable-geometry turbocharger and exhaust gas recirculation by optimization of model parameters for capturing non-linear system dynamics", Proc. of the IMechE, Part D: J. of Automobile Engineering, 225(7):960-986, 2011

[11] . E. Ffowcs Williams and X. Y. Huang, "Active stabilization of compressor surge”, Journal of Fluid Mechanics, 204:245-262, 61989.

[12] O. Leufvén, "Modeling for control of centrifugal compressors", $\mathrm{PhD}$ thesis 1516, Linköping University, 2013.

[13] J. P. Jensen, A.F. Kristensen, S.C. Sorenson, N. Houbak, and E. Hendricks, "Mean value modeling of a small turbocharged diesel engine”, In SAE World Congr., Techn. Paper 910070, Feb 1991.

114] P. Andersson, "Air Charge Estimation in Turbocharged Spark Ignition Engines", $\mathrm{PhD}$ thesis 989, Linköping University, 2005.

[15] H. Tu, H. Chen, "Modeling of a Compressor's Performance Map by Fitting Function Methodology", Advanced Materials Research Vols. 779-780 (2013) pp 1194-1198.

[16] http://mathworld.wolfram.com/Erfc.html 